\title{
Downregulation of matrix metalloproteinase-19 induced by respiratory syncytial viral infection affects the interaction between epithelial cells and fibroblasts
}

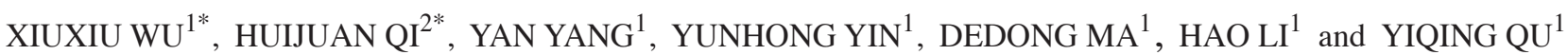 \\ ${ }^{1}$ Department of Respiratory Medicine, Qilu Hospital of Shandong University, Jinan, Shandong 250012; \\ ${ }^{2}$ Intensive Care Unit, The People's Hospital of Yucheng, Yucheng, Shandong 251200, P.R. China
}

Received December 14, 2014; Accepted October 5, 2015

DOI: $10.3892 / \mathrm{mmr} .2015 .4518$

\begin{abstract}
The present study was designed to examine the expression and function of matrix metalloproteinase-19 (MMP-19), which is downregulated following respiratory syncytial virus (RSV) infection. The diverse expression levels of MMP were examined using a designed cDNA expression array. The expression and secretion of MMP-19 was examined using reverse transcription-quantitative polymerase chain reaction (RT-qPCR) analysis and ELISA, respectively. The proliferation of epithelial cells and lung fibroblasts were examined using flow cytometry. The epithelial-mesenchymal transition (EMT) was also examined by performing western blot and RT-qPCR analyses. The results of the cDNA assay showed that infection with RSV resulted in the abnormal expression of certain metalloproteinases. Among these, the expression of MMP-19 decreased 3 and 7 days following infection. By using flow cytometric, western blot and RT-qPCR analyses, the present study demonstrated that the downregulation of MMP-19 inhibited the proliferation of epithelial cells, promoted the EMT and induced the proliferation of lung fibroblasts. Taken together, the findings of the present study suggested that the downregulation of MMP-19 following RSV infection may be associated with the development of airway hyper-responsiveness.
\end{abstract}

Correspondence to: Professor Yiqing Qu, Department of Respiratory Medicine, Qilu Hospital of Shandong University, 107 Wenhua Xi Road, Jinan, Shandong 250012, P.R. China

E-mail: quyiqing88@126.com

*Contributed equally

Key words: airway hyper-responsiveness, respiratory syncytial virus, matrix metalloproteinase-19, bronchial epithelial cells, epithelial-mesenchymal transition, lung fibroblast

\section{Introduction}

Respiratory syncytial virus (RSV) has become increasingly recognized as an important pathogen in pediatric viral bronchiolitis and pneumonia, and also causes severe respiratory infection in immunocompromised adults and the elderly (1). A possible link between RSV infection and asthma has been suggested in early childhood, and in subsequent manifestations of atopy and persistent asthma $(2,3)$. However, the mechanisms by which RSV may be involved in the development of post-bronchiolitis asthma and allergy remain to be fully elucidated.

The airway epithelium is central in initiating pulmonary inflammation, particularly in the case of RSV, as this virus productively replicates only in the respiratory mucosa $(4,5)$. Enzymes involved in degradation of the extracellular matrix, which have a number of important physiological effects, including remodeling of the extracellular matrix, facilitating cell migration, cleaving cytokines and activating defensins, may be important in initiating pulmonary diseases $(6,7)$. Reports from clinical investigations and animal models have shown that abnormal metalloproteinase causes matrix breakdown in patients with asthma $(8,9)$, suggesting that RSV infection may result in abnormality of the activities of certain metalloproteinases and trigger lung remodeling.

Whether the role of RSV in the pathogenesis of airway hyper-responsiveness is associated with abnormalities in the expression levels of metalloproteinase remains to be elucidated. In the present study, the diverse expression of metalloproteinases in RSV-infected 16-HBE human bronchial epithelial cells were screened using a cDNA microarray. Reverse transcription-quantitative polymerase chain reaction (RT-qPCR) analysis and ELISA were also used to further identify abnormalities in the expression of MMP-19. The correlation between the expression of MMP-19, the proliferation of epithelial cells and fibroblasts, and epithelial-mesenchymal transition (EMT) were also examined.

\section{Materials and methods}

Preparation of the RSV. The A2 strain of human RSV was propagated in a HeLa cell monolayer $\left(1 \times 10^{6}\right.$ cells at $90 \%$ confluence), both from the Research Institute of Virology 
(Wuhan, China) at $37^{\circ} \mathrm{C}$ in $5 \% \mathrm{CO}_{2}$ with $2 \%$ heat-inactivated fetal bovine serum (FBS; Gibco; Thermo Fisher Scientific, Inc., Waltham, MA, USA). At maximum cytopathic effect (large quantity of syncytia and residue of scattered islands of cells), the cells were repeatedly frozen and thawed three tomes to facilitate rupture of the cells. Subsequently, the supernatants were harvested and cellular debris was removed by centrifugation. The resulting RSV viral suspension was purified by centrifugation at $1,000 \mathrm{xg}$ for $15 \mathrm{~min}$ at $4^{\circ} \mathrm{C}$, filtered through a $0.22 \mu \mathrm{m}$ filter, aliquoted and stored at $-80^{\circ} \mathrm{C}$ until use. The viral titre was determined using a plaque assay.

Cell culture and RSV infection. The 16HBE human bronchial epithelial cells were cultured in Dulbecco's modified Eagle's medium combined with F12 (1:1; Cyclone, Logan, UT, USA) at $37^{\circ} \mathrm{C}$ in $5 \% \mathrm{CO}_{2}$ with $10 \% \mathrm{FBS}$. Following 2 days in culture, the cells at $90 \%$ confluence were infected with RSV at a multiplicity of infection (MOI) of 0.01 . The infected cells were collected after 3 days, when the cells exhibited a healthy cell monolayer morphology (10), and after 7 days, when a number of small syncytia began to form. In addition, a separate group of $16 \mathrm{HBE}$ cells were treated using the same procedure, but with uninfected HeLa cell lysate, and were used as a mock control group. RSV persistence was verified and monitored using an RSV Real-time PCR kit (Huayin Medicine Biotechnology Co., Ltd., Huayin, China). According to the manufacturer's protocol, the samples were considered negative for RSV when the quantification cycle $\left(C_{t}\right)$ value was $>32.0$. Samples with a $C_{t}$ value $\leq 28.9$ were considered positive for RSV.

Examination of the expression spectrum of metalloproteinase. The gene expression array was established by selecting all 84 known metalloproteinases, negative control (PUC18DNA and blank) and the housekeeping genes ( $\beta$-actin, GAPDH, cylcophilllin A and ribose body protein L13a) from a region of the whole chip. Shanghai Kangcheng Biological Technology Co., Ltd (Guangzhou, China) assisted with the establishment of the cDNA assay and the subsequent examination. cDNA were obtained from the cells by reverse transcription, labeling was performed with fluorescence at the 3 ' end, and the biotintylated cDNAs were hybridized to the designed metalloproteinase chip. The results were scanned using a GenePix 4000B chip scanner (Molecular Devices, Sunnyvale, CA, USA) and transformed into fluorescence signal intensity. The primary data were initially subtracted from the background value, and were subsequently adjusted by the housekeeping genes.

$R T-q P C R$. Total RNA was extracted from the $16 \mathrm{HBE}$ cells using TRIzol reagent, and reverse transcription was performed using a QuantiTect Reverse Transcription kit (cat. no. 205311; Takara Biotechnology, Co., Ltd., Dalian, China). qPCR was performed using an ABI Prism 7000 Sequence Detection system and software (Applied Biosystems; Thermo Fisher Scientific, Inc.) in a final volume of $50 \mu \mathrm{l}$ containing $2 \mu \mathrm{l}$ of cDNA synthesized from the RT reaction, 5 pmol of each primer, $25 \mu \mathrm{l}$ of SYBR Green Master Mix (Applied Biosystems; Thermo Fisher Scientific, Inc.), and $23 \mu \mathrm{l}$ of water. The amplification parameters included an initial $95^{\circ} \mathrm{C}$ for $5 \mathrm{~min}$, followed by 20 cycles of $95^{\circ} \mathrm{C}$ for $30 \mathrm{sec}$ and $60^{\circ} \mathrm{C}$ for $30 \mathrm{sec}$ The primers (Takara Biotechnology Co., Ltd, Dalian, China) used were as follows:
MMP-19, forward 5'-GTTGGGCTCTTATTGACGG-3' and reverse 5'-GAGAAGGCAAGGCTGGAA-3' (295 bp); E-cadherin, forward 5'-TCATAACCCACAGA TCCATT-3' and reverse 5'-CCAGGCGTAGACCAAGAA-3, (37bp); N-cadherin,forward 5'-ATCCTACTGGACGGTTCG-3' and reverse 5'-TTGGCTAATGGCACTTGA-3' (139 bp); and GAPDH forward 5'-CCACTCCTCCACCTTTGAC-3' and reverse 5'-ACCCTGTTGCTGTAGCCA-3'. Normalization of the RNA expression data was achieved by comparing the gray values of the target RNA with that of human GAPDH for each run. The PCR amplification products were sequenced following T-A cloning with a TOPO ${ }^{\circledR}$ TA cloning kit (Invitrogen, Thermo Fisher Scientific, Inc.) to direcly ligate the PCR products, to verify the specificity. Quantitative analysis of target gene expression data was based on the $2^{-\Delta \mathrm{Ct}}$ method (11).

Determination of the secretion of MMP-19 using ELISA. The supernatants of the mock-infected and RSV-infected cultures were collected on day 3 and day 7 following infection. Subsequently, ELISA was performed using an MMP-19 ELISA kit [cat no. YY(bio)-elisa-014490; R\&D Systems, Inc., Minneapolis, MN, USA], according to the manufacturer's protocol. Briefly, the cellular supernatants were centrifuged for $5 \mathrm{~min}$ at $500 \mathrm{x} \mathrm{g}$. The total supernatants or control samples $(100 \mu \mathrm{l})$ were added to a 96-well plate and incubated for $2 \mathrm{~h}$ at $37^{\circ} \mathrm{C}$. Following aspiration, the samples were incubated with $100 \mu \mathrm{l}$ Detection Reagent A for $1 \mathrm{~h}$ at $37^{\circ} \mathrm{C}$. Following washing three times with washing buffer, the Detection Reagent B was added and incubated for $30 \mathrm{~min}$ at $37^{\circ} \mathrm{C}$. Then, the samples were washed 5 times and $90 \mu 1$ Substrate Solution was added and incubated for $20 \mathrm{~min}$ at $37^{\circ} \mathrm{C}$. Subsequently $50 \mu 1$ Stop Solution was added to terminate the reaction. The $450 \mathrm{~nm}$ absorbance was determined using a microplate reader (Bio-Rad Laboratories, Inc., Hercules, CA, USA). Each sample was repeated three times. The minimum detectable dose of MMP-19 was $0.01 \mathrm{ng} / \mathrm{ml}$.

Construction of recombinant vectors. Fragments encoding the full coding sequence of MMP-19, containing a flag insert following ATG, were synthesized by GenScript Co., Ltd. (Nanjing, China) and cloned into the BamHI and XhoI sites of the pcDNA3.1(+) plasmid to construct pcDNA/MMP-19. The constructed plasmids were verified by restriction enzyme mapping, involving the double digestion with BamHI and XhoI (Invitrogen) to visualize the desired bands at 5.1 and $1.8 \mathrm{~kb}$, and direct DNA sequencing using T7 and sp6 primers. To generate MMP-19 small interfering (si)RNA expression constructs, three siRNA sequences were cloned into the site of a pGCU6/Neo/RFP vector to construct pGCU6/MMP-19siRNA. The most effective silenced plasmid (siRNA, ag CUCGUACUGUUCCAAUACUuu, was selected for use in the subsequent investigations.

Transfection and selection of recombinant plasmids. The $16 \mathrm{HBE}$ cells were seeded into six-well plates at a density of $5 \times 10^{5}$ cells per well. The recombinant plasmid DNA $(4 \mu \mathrm{g})$ and $8 \mu \mathrm{l}$ X-treme GENE HP DNA Transfection Reagent (Roche Diagnostics GmbH, Mannheim, Germany) were mixed with $200 \mu \mathrm{l}$ medium without antibiotics or FBS, and incubated at room temperature for $10 \mathrm{~min}$. Without removing the growth 
medium, this mixture was added to the $16 \mathrm{HBE}$ cells. pcDNA 3.1(+) and pGCU6/Neo/RFP were used for vector controls of the overexpressed and silenced plasmid, respectively. After $24 \mathrm{hr}$, the plasmids were selected with G418 (Ceresco, USA) at $1,000 \mathrm{mg} \mathrm{ml}^{-1}$ and subsequently cultured with G418 at $200 \mathrm{mg} \mathrm{ml}^{-1}$.

Measurement of cell cycle of using flow cytometry. Following treatment, the cells were fixed in cold $70 \%$ ethanol and stored at $-20^{\circ} \mathrm{C}$ overnight. The fixed cells were washed twice with PBS, stained with propidium iodide (Sigma-Aldrich, St. Louis, MO, USA) solution $(50 \mu \mathrm{g} / \mathrm{ml})$ for $1 \mathrm{~h}$ and treated with a ribonuclease A solution (20 $\mu \mathrm{g} / \mathrm{ml}$; Sigma-Aldrich) for $30 \mathrm{~min}$. Flow cytometry (BD Accuri C6; BD Accuri Cytometers, Ann Arbor, MI, USA) was then performed to examine the cell cycle.

Western blot analysis. The mock- and RSV-infected 16HBE cells were lysed in protease inhibitor cocktail solution (Roche Diagnostics). The cell lysates were quantified using spectrophotometery (BioSpectometer; Eppendorf, Hamburg, Germany) and $60 \mu \mathrm{g}$ were separated by SDS-PAGE (10\%; Bio-Rad Laboratories, Inc.) and transferred onto a nitrocellulose membrane (EMD Millipore, Billerica, MA, USA). The membrane was blocked with $3 \%$ bovine serum albumin in PBS for $2 \mathrm{~h}$, followed by incubation with 1:250 dilutions of polyclonal rabbit-anti-human $\mathrm{N}$-cadherin and E-cadherin antibodies (Abcam; cat. nos. 15148 and 12221) and polyclonal goat-anti human MMP-19 antibody [cat no. AF6790; R\&D Systems, Inc., Minneapolis, MN, USA] at $4^{\circ} \mathrm{C}$ overnight. The membrane was then incubated with horseradish peroxidase-conjugated goat anti-rabbit secondary antibody (1:5,000; EMD Millipore) for $2 \mathrm{~h}$ at room temperature. Detection was performed using an enhanced bioluminescence system (Gene Co., Ltd., Hong Kong, China). The bands were analyzed using ImageJ software (National Institutes of Health, Bethesda, MA, USA)

Co-culture of $16 \mathrm{HBE}$ cells with human lung fibroblasts (HLFs). In the co-culture experiments, the 16HBE cells were seeded at the bottom of a 24-well plate at a density of $10^{5}$ cells (1 $\mathrm{ml} /$ well) with normal growth media, and were grown to $\sim 50 \%$ confluence. The HLFs were seeded into Transwell chambers (Corning Inc., Corning, NY, USA) at a density of $2 \times 10^{4}$ with normal growth media for $12 \mathrm{~h}$ at $37^{\circ} \mathrm{C}$, following which the medium was replaced with $1 \mathrm{ml}$ medium containing $1 \%$ serum for another $12 \mathrm{~h}$. Subsequently, the Transwell chambers were placed in the wells with the 16HBE cells for co-culture.

Statistical analysis. Data are expressed as the mean \pm standard error of the mean. Statistical significances were assessed using either the variance among multiple samples or $q$-test between groups. $\mathrm{P}<0.05$ was considered to indicate a statistically significant difference. Analysis was performed using SPSS 11.0 for windows (SPSS, Inc., Chicago, IL, USA).

\section{Results}

Expression of MMP-19 gradually decreases in RSV-infected $16 H B E$ cells. The chip results were scanned and analyzed using software packages. The genes, in which expression levels
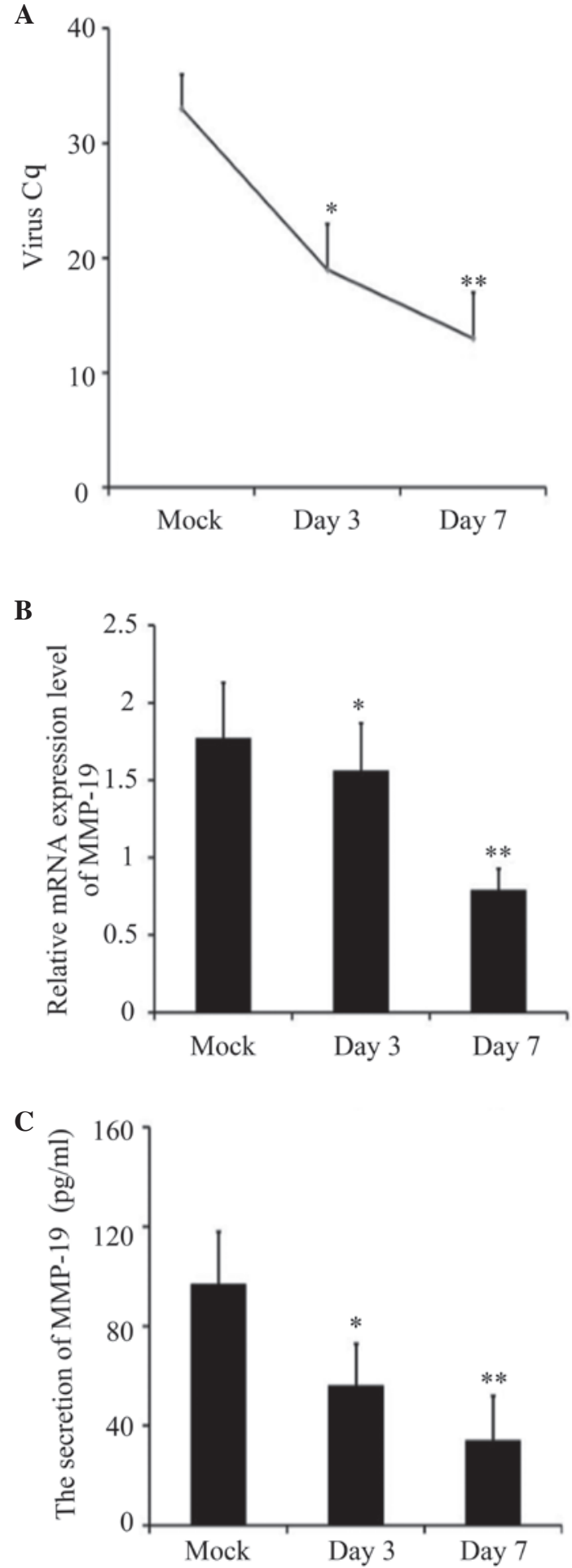

Figure 1. Expression levels of RSV and MMP-19. (A) Quantification cycle values of the RSV were examined using RT-qPCR. The mRNA expression of RSV increased significantly between day 3 and day 7. (B) mRNA expression levels of MMP-19 were evaluated using RT-qPCR. (C) Secretion of MMP-19 was evaluated using ELISA. The expression and release of MMP-19 decreased gradually between day 3 and day 7 . Data are expressed as the mean \pm standard error of the mean. ${ }^{*} \mathrm{P}<0.05$ and ${ }^{* *} \mathrm{P}<0.01$, vs. mock control. MMP-19 matrix metalloproteinase-19; RSV, respiratory syncytial virus. RT-qPCR, reverse transcription-quantitative polymerase chain reaction.

were increased more than twice were regarded as upregulated genes, and those in which expression levels decreased by $>0.5$ times were regarded as downregulated genes. The results 

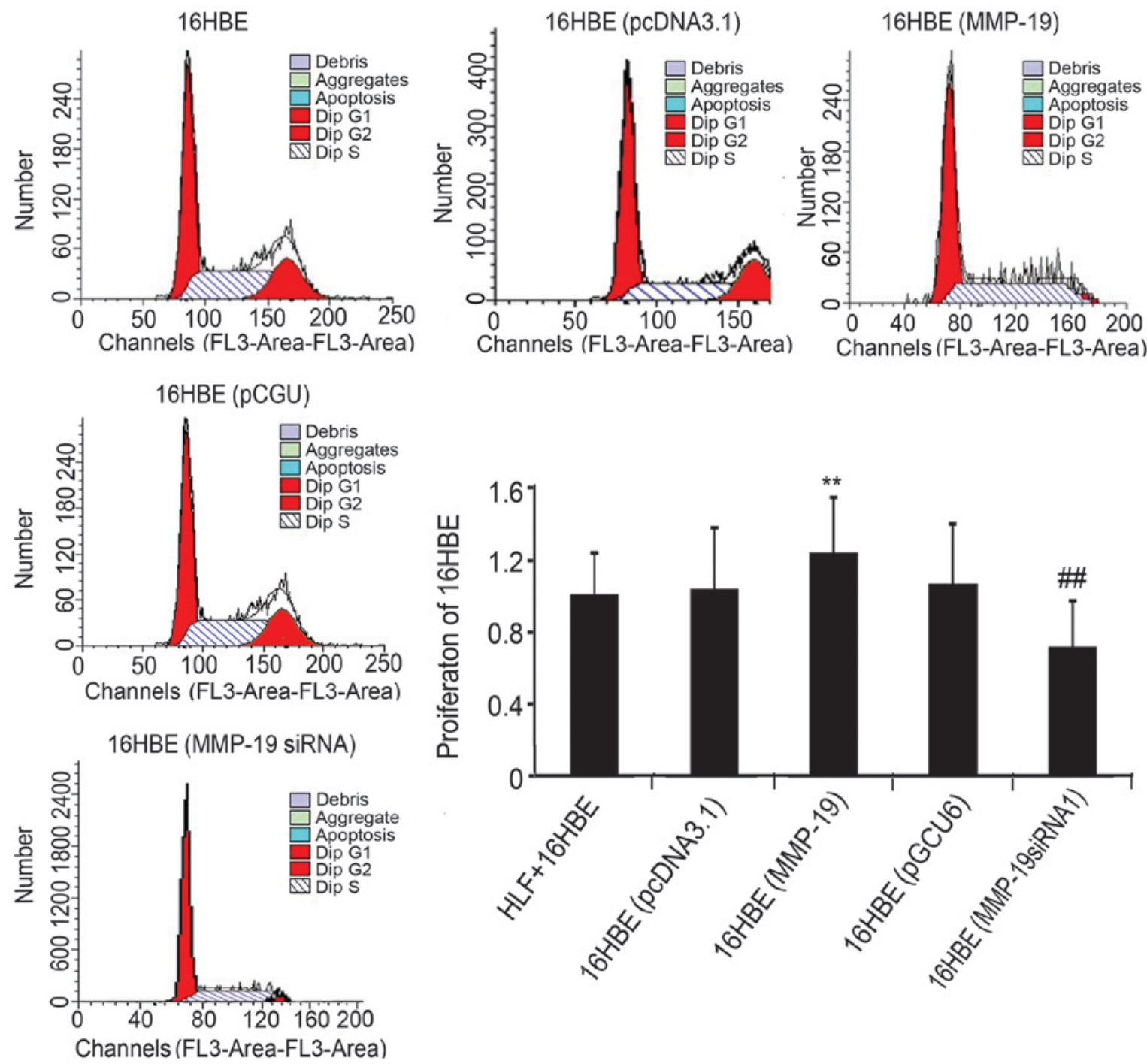

Figure 2. Effects of MMP-19 on the proliferation of 16HBE cells. Compared with the pcDNA3.1-transfected group, proliferation of the 16HBE cells increased by $9.48 \%$ in the MMP-19-overexpressed group. Compared with the pGCU6-transfected group, proliferation of the cells decreased by $13.27 \%$ in the MMP-19-silenced group. Data are expressed as the mean \pm standard error of the mean. ${ }^{* *} \mathrm{P}<0.01$ vs. the pcDNA3.1-transfected group; ${ }^{\sharp \#} \mathrm{P}<0.01$ vs. the pGCU6/Neo/RFP-transfected group. MMP-19 matrix metalloproteinase-19; siRNA, small interfering RNA.

showed that, compared with the mock-infected control cells, there were five upregulated genes, including MMP2, MMP-15, a disintegrin and metalloprotease domain (ADAM)9, ADAM33 and ADAMTS2, and nine downregulated genes, including MMP-7, MMP-17, MMP-19, uPA, TIMP-1, TIMP2, ADAMTS1, ADAMTS10 and ADAM10. Among these, MMP-19 was downregulated 0.65 and 1.33 times at day 3 and day 7 , respectively. The present study subsequently examined the expression and function of MMP-19 in the cultured 16HBE cells.

mRNA expression and secretion of MMP-19 in 16HBE cells. To verify the effects of RSV on the expression of MMP-19, mRNA from obtained from the mock- and RSV-infected 16HBE cells and analyzed using RT-qPCR 3 and 7 days following infection. The results showed that the mRNA expression of MMP-19 decreased significantly on days 3 and 7 following infection (Fig. 1). Enzyme immunoassay analyses of the culture supernatants also demonstrated that the expression of MMP-19 decreased on days 3 and 7, whereas the expression of MMP-19 in the control cells remained unchanged, as shown in Fig. 1.

Downregulation of MMP-19 inhibits cell cycle in $16 \mathrm{HBE}$ cells. To further examine the role of MMP-19 on the proliferation of $16 \mathrm{HBE}$ cells, the present study examined the cell cycle of normal, pcDNA3.1-transfected, pcDNA/MMP-19-transfected, pGCU6/Neo/RFP-transfected and pGCU6/MMP-19siRNA-transfected 16HBE cells using flow cytometry. The results revealed that, compared with the corresponding empty vector-transfected groups, the percentage of cells in the $(\mathrm{G} 2+\mathrm{S}) / \mathrm{G} 1$ phase increased by $9.48 \%$ in the MMP-19-overexpressing group, and decreased by $13.27 \%$ in the MMP-19-silenced group (Fig. 2).

Downregulation of MMP-19 promotes cadherin switching in $16 H B E$ cells. Proteins were collected from the cultured cells in the five treatment groups and evaluated using immunoblotting to determine the expression levels of E-cadherin and N-cadherin. The results showed that the expression of E-cadherin increased 
A

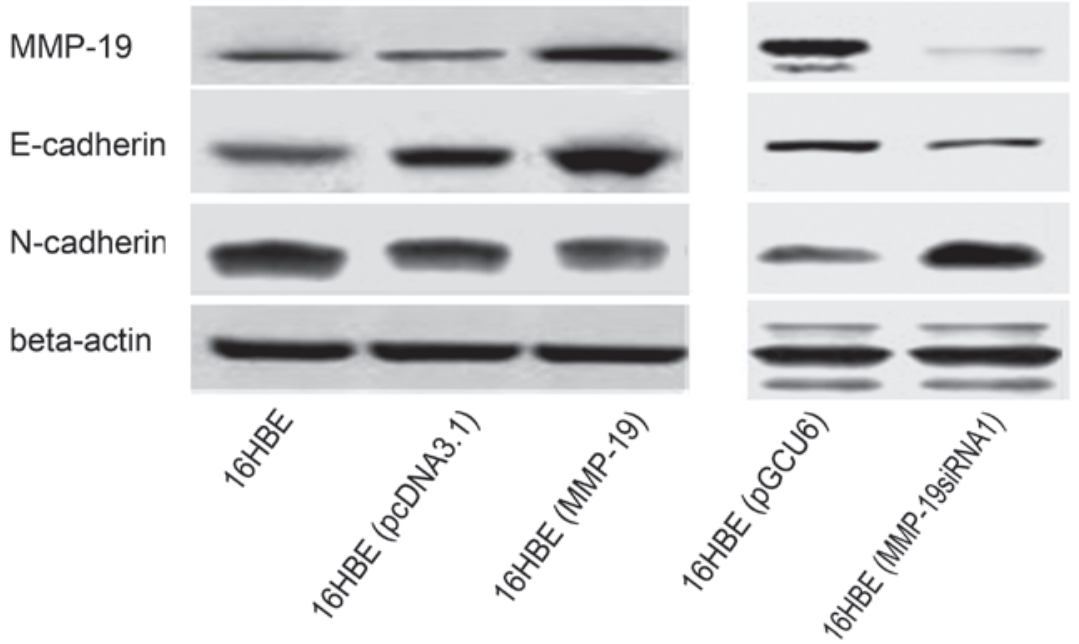

B

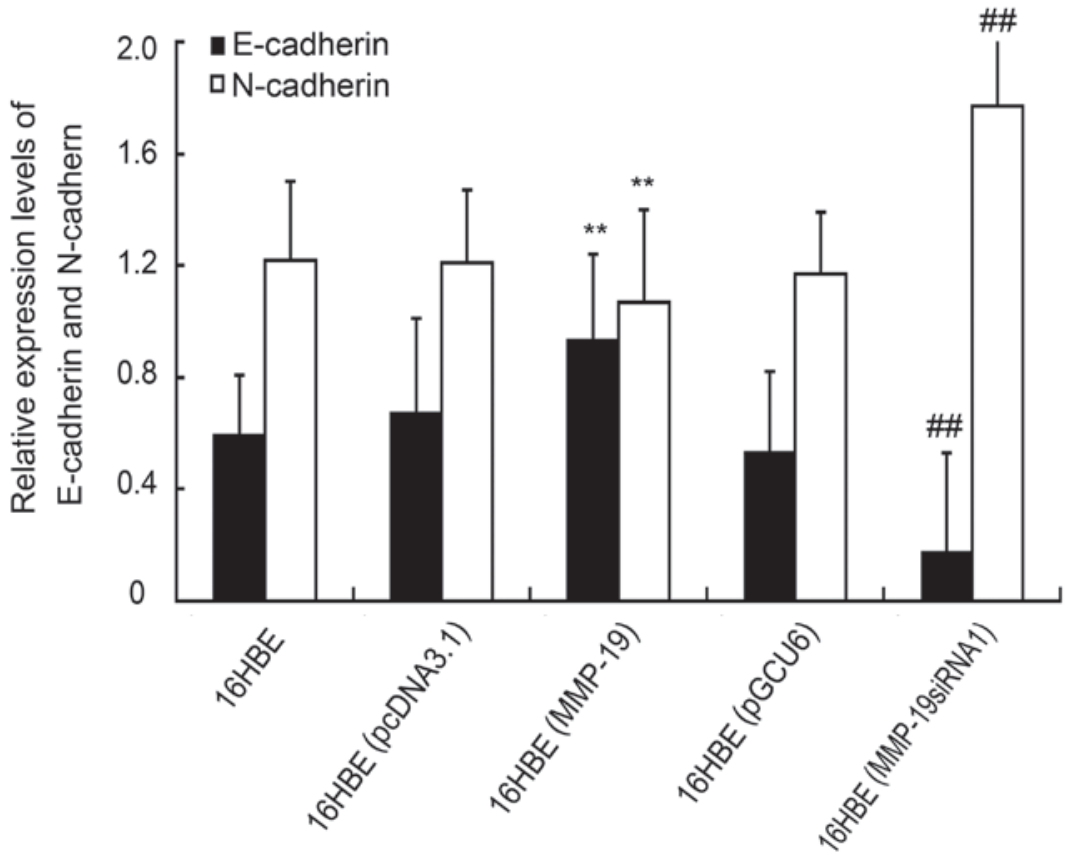

Figure 3. Cadherin switching is induced by downregulation of the expression of MMP-19. The protein and mRNA expression levels of MMP-19, E-cadherin and $\mathrm{N}$-cadherin were examined using (A) western blot analysis and (B) reverse transcription-quantitative polymerase chain reaction analysis, respectively. Cadherin switching was observed in the MMP-19-silenced (pGCU) group of cells. Data are expressed as the mean \pm standard error of the mean ( $\mathrm{n}=4-5$ per group). ${ }^{* *} \mathrm{P}<0.01$, vs. the pcDNA3.1-transfected group; ${ }^{\# \prime} \mathrm{P}<0.01$, vs. the pGCU6-transfected group. MMP-19 matrix metalloproteinase-19; siRNA, small interfering RNA.

in the MMP-19-overexpressed cells, compared with the pcDNA3.1(+)-transfected cells. Cadherin switching, indicative of EMT, was observed in the MMP-19-silenced cells, compared with the pGCU6/Neo/RFP-transfected group (Fig. 3).

Downregulation of MMP-19 promotes the proliferation of $H L F s$. In order to examine the effect of the downregulation of MMP-19 in 16HBE cells on the proliferation of lung fibroblasts under co-culture conditions, the proliferative activity of lung fibroblasts were determined using flow cytometry. The results (Fig. 4) demonstrated that the proliferation of the HLFs co-cultured with the MMP-19-overexpressing 16HBE cells were lower than that of the pcDNA3.1(+)-transfected group $(\mathrm{P}<0.01)$. However, the proliferation of the HLFs co-cultured with MMP-19-silenced 16HBE increased significantly, compared with that of the pGCU6/Neo/RFP-transfected cells $(\mathrm{P}<0.01)$.

\section{Discussion}

The formation of airway hyper-responsiveness is a type of response to airway epithelial injury (12). A number of clinical and basic investigations have confirmed that RSV in early childhood is an important risk factor for subsequent airway hyper-responsiveness; however, the underlying mechanism remains to be fully elucidated. Persistent RSV infections have been established in several human and animal epithelial cell lines (10); however, whether human epithelial cells of bronchial origin can permit viral persistent infections in vitro is an area of debate. The present study aimed to determine whether RSV is able to infect the 16HBE human bronchial epithelial cell line over multiple generations. The results showed that, when RSV at an MOI of 0.01 was used to infect the $16 \mathrm{HBE}$ cells, RSV survived to four generations. 

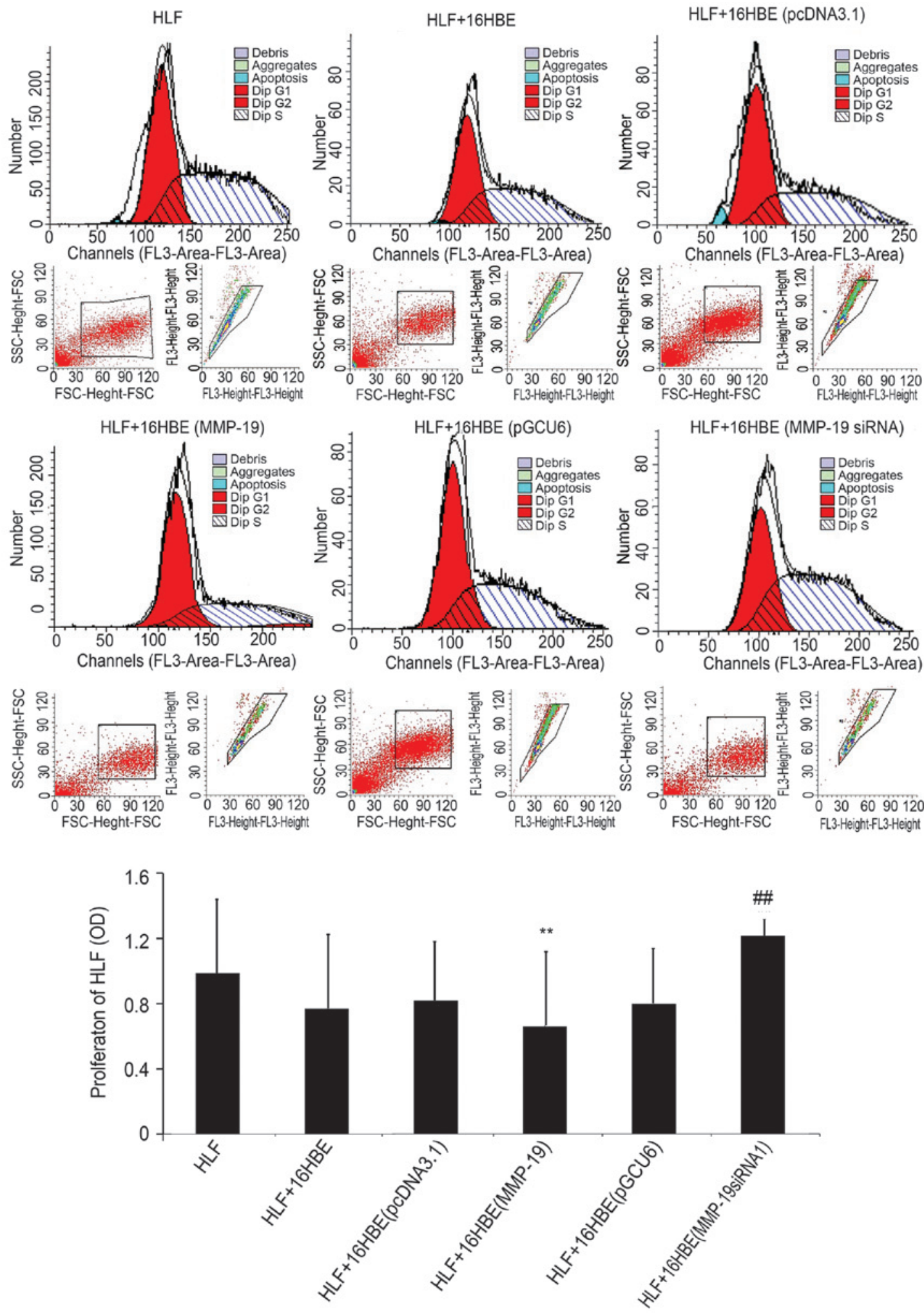

Figure 4. MMP-19 overexpression in 16HBE cells decreases the proliferation of HLFs. Proliferation of HLFs decreased following co-culture with MMP-19-overexpressed 16-HBE cells, compared with HLFs co-cultured with pcDNA3.1-transfected 16-HBEcells. However, the proliferation of the HLFs increased following co-culture with MMP-19-silenced 16-HBE cells, compared with the pGCU6-transfected 16-HBE cells. ${ }^{* *} \mathrm{P}<0.01$ vs. the pcDNA3.1-transfected group; ${ }^{\# \#} \mathrm{P}<0.01$ vs. the pGCU6/Neo/RFP-transfected group. MMP-19 matrix metalloproteinase-19; HLF, human lung fibroblast; siRNA, small interfering RNA; OD, optical density.

The establishment of an in vitro model of infected human bronchial epithelial cells, which persists for four generations prior to death, provides a novel system for characterizing persistent RSV mechanisms.
MMP-19 was fist cloned from a human liver cDNA library in 1997 (13), which has been shown to degrade a variety of substrates of the extracellular matrix and the basement membrane, including collagen type 4 , large tenascin-C 
isoform, fibronectin, type 1 gelatin, laminin-5, nidogen-1, aggrecan and cartilage oligomeric matrix protein (14-16). Brauer et al (17) reported that MMP-19 digests plasminogen to produce fragments with bioactivities of angiostatin, which inhibit proliferation and capillary-growth of endothelial cells and implicate MMP-19 in vascular remodeling and angiogenesis. Gueders et al (18) showed that MMP-19 deficiency promotes the accumulation of tenascin-C and allergen-induced airway inflammation. The present study, which investigated the cellular levels of MMP-19, revealed that human RSV infection in cultured 16HBE cells resulted in downregulated expression levels of MMP-19.

EMT is a mechanism, which may account for the accumulation of subepithelial mesenchymal cells, thereby contributing to increased contractile cell mass and airway hyperresponsiveness. EMT is predominantly characterized by the loss of epithelial markers, including E-cadherin, and the acquisition of mesenchymal markers including vimentin and $\mathrm{N}$-cadherin (19). A previous study involving a mouse model of chronic house dust mite-driven allergic airway inflammation demonstrated the capacity of airway epithelial cells to acquire mesenchymal characteristics under these conditions (20). The results of the present study demonstrated that downregulation in the expression levels of MMP-19 induced loss of the characteristic airway epithelial cell marker.

Under normal conditions, reciprocal inhibition in proliferation exists between bronchial epithelial cells and lung fibroblasts, which is essential for maintenance of homeostasis in the airway architecture. In this state, bronchial epithelial cells inhibit the proliferation of lung fibroblasts. However, downregulation of MMP-19 in the 16HBE bronchial epithelial cells promoted the proliferation of lung fibroblasts, indicating the activation of lung fibroblasts following RSV infection.

In conclusion, the mechanism underlying the pathogenesis of RSV in airway hyper-responsiveness may include abnormal expression levels of certain metalloproteinases to inhibit the function of epithelial cells and assist in the proliferation and migration of lung fibroblasts. The present study is the first, to the best of our knowledge, to report that the expression of MMP-19 decreased in cultured 16HBE cells following RSV infection, which provides an experimental basis for further elucidation of the mechanism of RSV-induced airway hyperresponsiveness.

\section{Acknowledgements}

This study was supported by a grant from the Scientific and Technological Research Project of Shandong Province (grant. no. 2007GG3002008).

\section{References}

1. Backman K, Piippo-Savolainen E, Ollikainen H, Koskela H and Korppi M: Adults face increased asthma risk after infant RSV bronchiolitis and reduced respiratory health-related quality of life after RSV pneumonia. Acta Paediatr 103: 850-855, 2014.

2. Simões EA, Carbonell-Estrany X, Rieger $\mathrm{CH}$, Mitchell I, Fredrick L and Groothuis JR; Palivizumab Long-Term Respiratory Outcomes Study Group: The effect of respiratory syncytial virus on subsequent recurrent wheezing in atopic and nonatopic children. J Allergy Clin Immunol 126: 256-262, 2010.
3. Silver E, Yin-DeClue H, Schechtman KB, Grayson MH, Bacharier LB and Castro M: Lower levels of plasmacytoid dendritic cells in peripheral blood are associated with a diagnosis of asthma 6 yr after severe respiratory syncytial virus bronchiolitis. Pediatr Allergy Immunol 20: 471-476, 2009

4. Tan Y, Yang T, Liu S, Liu H, Xiang Y, Qu F, Li H and Qin X: Infection with respiratory syncytial virus alters peptidergic innervation in the lower airways of guinea-pigs. Exp Physiol 93: 1284-1291, 2008

5. Liu X, Qin X, Xiang Y, Liu H, Gao G, Qin L, Liu C and Qu X: Progressive changes in inflammatory and matrix adherence of bronchial epithelial cells with persistent respiratory syncytial virus (RSV) infection (progressive changes in RSV infection). Int J Mol Sci 14: 18024-18040, 2013.

6. Zariffard MR, Anastos K, French AL, Munyazesa E, Cohen M, Landay AL and Spear GT: Cleavage/alteration of interleukin-8 by matrix metalloproteinase- 9 in the female lower genital tract. PLoS One 10: e0116911, 2015.

7. Wilson CL, Schmidt AP, Pirilä E, Valore EV, Ferri N, Sorsa T, Ganz T and Parks WC: Differential Processing of \{alpha\}- and \{beta\}-Defensin Precursors by Matrix Metalloproteinase-7 (MMP-7). J Biol Chem 284: 8301-8311, 2009.

8. Barbaro MP, Spanevello A, Palladino GP, Salerno FG, Lacedonia D and Carpagnano GE: Exhaled matrix metalloproteinase-9 (MMP-9) in different biological phenotypes of asthma. Eur J Intern Med 25: 92-96, 2014.

9. Weitoft M, Andersson C, Andersson-Sjöland A, Tufvesson E, Bjermer L, Erjefält J and Westergren-Thorsson G: Controlled and uncontrolled asthma display distinct alveolar tissue matrix compositions. Respir Res 15: 67, 2014.

10. Liu X, Qin X, Xiang Y, Liu H, Gao G, Qin L, Liu C and Qu X: Progressive changes in inflammatory and matrix adherence of bronchial epithelial cells with persistent respiratory syncytial virus (RSV) infection (progressive changes in RSV infection). Int J Mol Sci 14: 18024-18040, 2013.

11. Dwivedi S, Goel A, Mandhani A, Khattri S, Sharma P, Misra S and Pant KK: Functional genetic variability at promoters of pro-(IL-18) and anti-(IL-10) inflammatory affects their mRNA expression and survival in prostate carcinoma patients: Five year follow-up study. Prostate 75: 1737-1746, 2015.

12. Tan YR, Yang T, Liu SP, Xiang Y, Qu F, Liu HJ and Qin XQ: Pulmonary peptidergic innervation remodeling and development of airway hyperresponsiveness induced by RSV persistent infection. Peptides 29: 47-56, 2008.

13. Pendás AM, Knäuper V, Puente XS, Llano E, Mattei MG, Apte S, Murphy $\mathrm{G}$ and López-Otín C: Identification and characterization of a novel human matrix metalloproteinase with unique structural characteristics, chromosomal location and tissue distribution. J Biol Chem 272: 4281-4286, 1997.

14. Stracke JO, Fosang AJ, Last K, Mercuri FA, Pendás AM, Llano E, Perris R, Di Cesare PE, Murphy G and Knäuper V: Matrix metalloproteinases 19 and 20 cleave aggrecan and cartilage oligomeric matrix protein (COMP). FEBS Lett 478: 52-56, 2000.

15. Sadowski T, Dietrich S, Koschinsky F, Ludwig A, Proksch E, Titz B and Sedlacek R: Matrix metalloproteinase 19 processes the laminin 5 gamma 2 chain and induces epithelial cell migration. Cell Mol Life Sci 62: 870-880, 2005.

16. Titz B, Dietrich S, Sadowski T, Beck C, Petersen A and Sedlacek R: Activity of MMP-19 inhibits capillary-like formation due to processing of nidogen-1. Cell Mol Life Sci 61: 1826-1833, 2004.

17. Brauer R, Beck IM, Roderfeld M, Roeb E and Sedlacek R: Matrix metalloproteinase-19 inhibits growth of endothelial cells by generating angiostatin-like fragments from plasminogen. BMC Biochem 12: 38, 2011.

18. Gueders MM, Hirst SJ, Quesada-Calvo F, Paulissen G, Hacha J, Gilles C, Gosset P, Louis R, Foidart JM, Lopez-Otin C, et al: Matrix metalloproteinase-19 deficiency promotes tenascin- $\mathrm{C}$ accumulation and allergen-induced airway inflammation. Am J Respir Cell Mol Biol 43: 286-295, 2010.

19. Johnson JR, Nishioka M, Chakir J, Risse PA, Almaghlouth I, Bazarbashi AN, Plante S, Martin JG, Eidelman D and Hamid Q: IL-22 contributes to TGF- $\beta 1$-mediated epithelial-mesenchymal transition in asthmatic bronchial epithelial cells. Respir Res 14: 118,2013

20. Johnson JR, Roos A, Berg T, Nord M and Fuxe J: Chronic respiratory aeroallergen exposure in mice induces epithelial-mesenchymal transition in the large airways. PLoS One 6: e16175, 2011. 